Pacific Journal of Mathematics

A NEW LOOK AT SOME FAMILIAR SPACES OF 


\title{
A NEW LOOK AT SOME FAMILIAR SPACES OF INTERTWINING OPERATORS
}

\author{
RALPH Gellar AND LAvon PAGE
}

\begin{abstract}
When $T_{1}$ and $T_{2}$ are bounded operators on a Hilbert space, solutions to the equation $X T_{1}=T_{2} X$ are called intertwining operators for $T_{1}$ and $T_{2}$. Several familiar spaces of intertwining operators are examined and a new method is proposed for studying the duality relationship that frequently exists between the space of intertwining operators and its subspace of compact intertwining operators.
\end{abstract}

Evidently such operators form a Banach space if the usual operator norm is employed. We denote this space by $\mathscr{S}\left(T_{1}, T_{2}\right)$.

Such spaces of intertwining operators are abundantly present in the literature of operator theory. We cite these examples:

ExAMPLE 1. If $D$ is a diagonal operator with distinct, nonzero diagonal entries, then $\mathscr{F}(D, D)$, the commutant of $D$, is simply the familiar space of all diagonal matrices and is isomorphic to $\iota^{\infty}$.

EXAMPLE 2. In case $S$ is either the simple unilateral or bilateral shift, $\mathscr{F}(S, S)$ is the space of analytic Toeplitz operators or Laurent operators respectively.

EXAMPLE 3. Let $T$ be the restriction of the adjoint of the simple shift to an invariant subspace. In [5], Kriete, Moore, and Page studied $\mathscr{J}\left(T^{*}, T\right)$.

EXAMPLE 4. If $S$ is a unilateral shift (of any multiplicity), then $\mathscr{J}\left(S, S^{*}\right)$ is the space of all $S$-Hankel operators.

All of the above spaces of intertwining operators have been studied in depth. The phenomenon which motivates our investigation is that in Examples 1,3, and 4 the space of intertwining operators in question is isomorphic to the second dual space of its compact part, i.e., to the second dual of the space obtained by intersecting $\mathscr{J}\left(T_{1}, T_{2}\right)$ with the space of compact operators on $\mathscr{H}$.

By way of contrast, there are no compact operators at all in the spaces of Example 2.

That the spaces of Examples 1,3, and 4 above have the stated biduality property is seen by representing the spaces of intertwining operators under study as familiar function spaces (or quotient spaces of such), and then determining the duality properties of said function 
spaces. With Example 1, this is elementary. The biduality in Example 4 is due to Kriete, Moore, and Page. The duality theorem for Hankel operators was obtained by Hevener [4] and Page [6].

Herein we describe an alternate procedure for examining the question, "When is $\mathscr{F}\left(T_{1}, T_{2}\right)$ the second dual of its compact part?" Our procedure is simply stated (see Theorem 1) and its abstract foundation is unsophisticated; yet it is adequate in investigating the above examples. We do this in Section II where we also use the technique to pass to more complex examples where the duality between a space and its compact part would be most difficult to establish by earlier methods.

I. General theory. Let $\mathscr{B}(\mathscr{C})$ and $\mathscr{C}(\mathscr{C})$ be the spaces of bounded operators and compact operators respectively on $\mathscr{H}$.

We recall the manner in which $\mathscr{B}(\mathscr{C})$ is identified as the second dual space of $\mathscr{C}(\mathscr{C})$. The space of trace-class operators on $\mathscr{P}$ is isomorphic to the dual space of $\mathscr{C}(\mathscr{H})$; under the isomorphism the trace-class operator $Q$ corresponds to the linear functional $T \rightarrow \operatorname{tr}(T Q)$ where "tr" denotes the trace. In a similar manner $\mathscr{B}(\mathscr{C})$ acts as the dual of the space of trace-class operators. (See Schatten [7, pp. 45-53].)

If $\mathscr{C}$ is a subset of $\mathscr{B}(\mathscr{C})$, then $\mathscr{C}^{-}$will denote the closure of $\mathscr{X}$ in the ultra-weak operator topology on $\mathscr{B}(\mathscr{H})$, i.e., the weakstar topology on $\mathscr{B}(\mathscr{X})$ as the dual of the space of trace-class operators, and $\mathscr{Z}^{\sim}$ will denote the closure of $\mathscr{Z}^{\prime}$ in the weak operator topology.

Proposition 1. If 2 is a closed subspace of $\mathscr{C}(\mathscr{H})$, then

(a) $\mathscr{X} \subseteq \mathscr{X}^{-} \subseteq \mathbb{Z}^{\sim}$, and

(b) $\mathscr{Z}^{-}$is precisely the set of all limits of bounded nets in $\mathscr{C}^{2}$ convergent in the weak operator topology.

Proof. To say that $A_{\alpha} \rightarrow A$ in the weak operator topology is precisely to say that $\operatorname{tr}\left(A_{\alpha} Q\right) \rightarrow \operatorname{tr}(A Q)$ for every operator $Q$ of rank one.

Assertion (b) is a consequence of the following two facts:

(1) The unit ball of $\mathscr{X}$ is weak* dense in the unit ball of its second dual $\mathscr{X}^{-}[3$, p. 424]. (See proof of Theorem 1 below.)

(2) A bounded net converges in the weak operator topology if and only if it converges in the ultra-weak operator topology.

The second assertion is also implicitly contained in [3, p. 512] as an exercise. We give a simple proof here for completeness.

Suppose $\left\{A_{\alpha}\right\}$ converges to $A$ in the weak operator topology, and that $\left\{A_{\alpha}\right\}$ is a bounded net. Then for any operator $Q$ of finite rank, 
it is easy to see that $\operatorname{tr}\left(A_{\alpha} Q\right) \rightarrow \operatorname{tr}(A Q)$. But the finite rank operators are dense in the space of trace-class operators, and the linear functionals $Q \rightarrow \operatorname{tr}\left(A_{\alpha} Q\right), Q$ in trace-class, are uniformly bounded. Hence $\operatorname{tr}\left(A_{\alpha} Q\right) \rightarrow \operatorname{tr}(A Q)$ for every $Q$ of trace-class, or $A_{\alpha} \rightarrow A$ in the ultraweak operator topology.

The following theorem is quite elementary in the abstract, but it will be of considerable use in analyzing the examples of Sections I and II.

Theorem 1. Let $T_{1}$ and $T_{2}$ be bounded operators on $\mathscr{H}$. Then $\mathscr{I}\left(T_{1}, T_{2}\right)$ is isometrically isomorphic to the second dual of its compact part provided that each operator in $\mathscr{J}\left(T_{1}, T_{2}\right)$ is the limit in the weak operator topology of a sequence of compact operators in $\mathscr{J}\left(T_{1}, T_{2}\right)$.

Proof. Let $\mathscr{C}\left(T_{1}, T_{2}\right)$ denote the compact part of $\mathscr{J}\left(T_{1}, T_{2}\right)$. In general we have

$$
\mathscr{C}\left(T_{1}, T_{2}\right) \subseteq \mathscr{C}\left(T_{1}, T_{2}\right)^{-} \subseteq \mathscr{C}\left(T_{1}, T_{2}\right)^{\sim} \subseteq \mathscr{F}\left(T_{1}, T_{2}\right)
$$

in light of Proposition 1(a).

Theorem 1 is a consequence of the following duality property of Banach spaces:

If $\mathscr{Y}$ is a Banach space, and $\mathscr{X}$ is a weak* closed subspace of $\mathscr{Y}^{* *}$, then there is a natural isomorphism that identifies $(\mathscr{P} \cap \mathscr{Y})^{* *}$ with the weak* closure of $\mathscr{X} \cap \mathscr{Y}$ in $\mathscr{Y}^{* *}$. The isomorphism arises as follows: Let $\mathscr{C}$ denote the annihilator of $\mathscr{X} \cap \mathscr{Y}$ in $\mathscr{Y}^{*}$. First note that the annihilator of $\mathscr{C l}$ in $\mathscr{Y}^{* *}$ is simply the weak* closure of $\mathscr{X} \cap \mathscr{Y}$ in $\mathscr{Y}^{* *}[1$, p. 53]. But the Hahn-Banach theorem provides natural isomorphisms between $(\mathscr{X} \cap \mathscr{Y})^{*}$ and $\mathscr{Y}^{*} / \mathscr{C l}$ and between $\left(\mathscr{Y}^{*} / \mathscr{M}\right)^{*}$ and the annihilator of $\mathscr{C}$ in $\mathscr{Y}^{* *}$ [2, p. 25-27].

From the preceding discussion it is apparent that $\mathscr{C}\left(T_{1}, T_{2}\right)^{* *}$ is isomorphic to $\mathscr{F}\left(T_{1}, T_{2}\right)$ if $\mathscr{C}\left(T_{1}, T_{2}\right)^{-}=\mathscr{F}\left(T_{1}, T_{2}\right)$. According to Proposition 1 this will be the case if and only if every operator in $\mathscr{F}\left(T_{1}, T_{2}\right)$ is the limit in the weak operator topology of a bounded net in $\mathscr{C}\left(T_{1}, T_{2}\right)$. But the very same limits can be reached through bounded nets as through sequences. This is true first because (by the uniform boundedness principle) weak operator convergent sequences must be bounded. Secondly, according to Proposition 1 the same bounded nets converge in the weak operator topology as in the ultraweak operator topology. But such nets can be replaced by convergent sequences, since the ultra-weak operator topology is metrizable on bounded sets [3, p. 426].

CoRollary. If $T$ is in $\mathscr{B}(\mathscr{H})$ then the commutant of $T$ is isometrically isomorphic to the second dual of the compact commutant of $T$ 
provided there exists a sequence of compact operators which commute with $T$ and converge in the weak operator topology to the identity.

Proof. If $\left\{X_{n}\right\}$ is a sequence of compact operators which commute with $T$ and converge in the weak operator topology to $I$, then for any operator $B$ that commutes with $T,\left\{X_{n} B\right\}$ converges to $B$ in the weak operator topology. Since $X_{n} B$ commutes with $T$ for each $n$, and since $X_{n} B$ is compact, the conclusion now follows from Theorem 1 .

II. Examples. In this section we explore several examples of spaces of intertwining operators that are amenable to study via Theorem 1.

\section{EXAMPLE 1. Diagonal operators.}

Let $D$ be a diagonal operator on $\mathscr{H}$ with distinct nonzero entries on the diagonal. It is easy to see that the commutant of $D$ is the set of diagonal operators and is isomorphic to $l^{\infty}$. The compact commutant of $D$ consists of those diagonal operators whose diagonal entries tend to zero, and hence the compact commutant is isomorphic to $c_{0}$, the space of complex sequences converging to zero. Clearly the identity is the weak-operator limit of a sequence of finite rank diagonal operators. Thus by the preceding corollary the second dual of the compact commutant of $D$ is naturally isomorphic to the commutant of $D$. This, of course, substantiates the well-known fact that $l^{\infty}$ is the second dual of $c_{0}$.

\section{EXAMPLE 2. Weighted Hankel operators.}

The following theorem provides a simple approach to the result that $\mathscr{J}\left(S, S^{*}\right)$ is isomorphic to $\mathscr{C}\left(S, S^{*}\right)^{* *}$ when $S$ is a simple unilateral shift, the case in which $\mathscr{F}\left(S, S^{*}\right)$ and $\mathscr{C}\left(S, S^{*}\right)$ are spaces of Hankel operators. Here we shall in fact allow $S$ to be a weighted shift. Let $\left\{e_{n}\right\}_{n=0}^{\infty}$ be an orthonormal basis for $\mathscr{C}$ and let $S: e_{n} \rightarrow a_{n+1} e_{n+1}$ where $\left\{a_{n}\right\}_{n=1}^{\infty}$ is a bounded sequence of complex numbers. We shall assume in addition that $\left\{a_{n}\right\}_{n=1}^{\infty}$ is bounded away from zero, so that $S$ has a bounded left inverse. Denote by $S^{+}$that left inverse of $S$ which sends $e_{0}$ to 0 . Clearly $S^{+}$sends $e_{n}$ to $a_{n}^{-1} e_{n-1}$ if $n \geqq 1$. The requirement that the weights be bounded away from zero is simply that zero not be in the approximate point spectrum of $S$.

THEOREM 2. Let $S$ be a weighted shift with weights bounded away from zero. Let $S^{+}$be the (bounded) left inverse of $S$ which sends $e_{0}$ to 0. Then $\mathscr{F}\left(S, S^{+}\right)$is isomorphic to $\mathscr{C}\left(S, S^{+}\right)^{* *}$. 
Proof. It is straightforward to check that an operator $X$ in $\mathscr{B}(\mathscr{C})$ satisfies $X S=S^{+} X$ if and only if the matrix of $X$ relative to the basis $\left\{e_{n}\right\}_{n=0}^{\infty}$ is of the form $\left[b_{i+j} w_{i} w_{j}^{-1}\right]$ where $w_{n}=\pi_{i=1}^{n} a_{i}$. It is reasonable to call such an operator a weighted Hankel operator. If $X$ is a weighted Hankel operator and $0<r<1$, then there exists a weighted Hankel operator $X_{r}$ on $\mathscr{H}$ whose matrix is $\left[r^{i+j} b_{i+j} w_{i} w_{j}^{-1}\right]$ and which satisfies $\left\|X_{r}\right\| \leqq\|X\|$. This is proved as follows: Let $f=\alpha_{0} e_{0}+\cdots+\alpha_{k} e_{k}$ and $g=\beta_{0} e_{0}+\cdots+\beta_{k} e_{k}$ where $k$ is a positive integer and $\left\{\alpha_{i}\right\}$ and $\left\{\beta_{i}\right\}$ are $k+1$-tuples of complex numbers. For $0<r<1$, let $f_{r}=\alpha_{0} e_{0}+r \alpha_{1} e_{1}+\cdots+r^{k} \alpha_{k} e_{k}$ and $g_{r}=\beta_{0} e+r \beta_{1} e_{1}+$ $\cdots r^{k} \beta_{k} e_{k}$. Straightforward arithmetic shows that $\left\langle X_{r} f, g\right\rangle=\left\langle X f_{r}, g_{r}\right\rangle$. Since $\left\|f_{r}\right\| \leqq\|f\|$ and $\left\|g_{r}\right\| \leqq\|g\|$, it then follows that $\left\|X_{r}\right\| \leqq\|X\|$.

Now for $0 \leqq i, j<\infty$ and $0<r<1,\left\langle X_{r} e_{i}, e_{j}\right\rangle=r^{i+j} b_{i+j} w_{i} w_{j}^{-1}$. As $r \rightarrow 1$, this tends to $b_{i+j} w_{i} w_{j}^{-1}$. Because $\left\{X_{r}\right\}$ is uniformly bounded, we can conclude that $\left\{X_{r}\right\}$ converges to $X$ in the weak operator topology.

For $0 \leqq i, j<\infty,\left|b_{i+j} w_{i} w_{j}^{-1}\right| \leqq\|X\|$. Thus the entries of the matrix of $X_{r}(0<r<1)$ are absolutely summable, and this implies the compactness of $X_{r}$.

Theorem 1 now shows that $\mathscr{J}\left(S, S^{+}\right)$is isomorphic to the second dual of $\mathscr{C}\left(S, S^{+}\right)$.

EXAMPle 3. Compressed shifts.

Operators of the form $S^{*} / \mathscr{C l}$ where $S$ is a shift and $\mathscr{C l}$ is an invariant subspace for $S^{*}$ are of extreme importance because of their use in canonical models. Kriete, Moore, and Page [5] showed that if $T_{1}$ and $T_{2}$ are operators of the above type where $S$ is a simple unilateral shift, then $\mathscr{S}\left(T_{1}, T_{2}\right)$ is the second dual of its compact part. In light of the model theory, such a result cannot possibly hold for shifts of infinite multiplicity.

The key technical result in [5] is that for any inner function $\delta$ in $H^{\infty}, H^{\infty} \cap \delta C$ is weak-star dense in $\mathscr{H}^{\infty}$, where $\mathscr{C}$ is the class of continuous functions on the unit circle. It can be shown that this technical lemma about the nature of $H^{\infty}$ is equivalent to the assertion that if $T_{1}$ and $T_{2}$ are operators of the form $S^{*} / \mathscr{C l}$ where $S$ is the simple unilateral shift and $\mathscr{C}$ is an invariant subspace for $S^{*}$, then each operator that intertwines $T_{1}$ and $T_{2}$ is the limit in the weak operator topology of a sequence of compact intertwining operators. Thus the biduality result of [5] may be viewed as a consequence of the technical lemma and Theorem 1.

ExAmPLE 4. Quasi-triangular and other miscellaneous classes of operators. 
Theorem 1 is generalized without change of proof by replacing $\mathscr{F}\left(T_{1}, T_{2}\right)$ by any weak operator closed subspace $\mathscr{P}$ of $\mathscr{B}(\mathscr{H})$ and replacing $\mathscr{C}\left(T_{1}, T_{2}\right)$ by $\mathscr{Z} \cap \mathscr{C}(\mathscr{H})$. The methods of Theorem 2 can also be generalized. Let $X$ be any bounded operator on $\mathscr{H}$ with matrix $\left[\alpha_{i j}\right]$ with respect to some orthonormal basis $\left\{e_{n}\right\}_{n=0}^{\infty}$. Define $X_{r}, 0<r<1$, to be the operator with matrix $\left[r^{i+j} a_{i j}\right]$ with respect to the same basis. Exactly as in Theorem 2 we can show that $\left\{X_{r}, r \rightarrow 1\right\}$ is a bounded net of compact operators converging to $X$ in the weak operator topology. We will then have proven the following theorem.

THEOREM 3. Let $\mathscr{X}$ be a subspace of $\mathscr{B}(\mathscr{C})$ which is closed in the weak operator topology. Suppose for each $X$ in there exists an orthonormal basis such that all the operators $X_{r}, 0<r<1$, also are in $\mathscr{X}$. Then $\mathscr{X}$ is isomorphic to the second dual space of $\mathscr{X} \cap \mathscr{C}(\mathscr{H})$.

For example, each of the following spaces-weighted shifts with respect to a fixed basis, the diagonal operators with respect to a fixed basis (mentioned earlier), quasi-triangular operators with respect to a fixed basis (associated matrix $\mathrm{a}_{i j}=0$ unless $i \leqq j+1$ ), operators with matrix satisfying $a_{j j}=-2 a_{j+1, j-1}$ (all $j$ ), etc. - is shown to be isomorphic to the second dual of its intersection with the compact operators. We note with glee that we are assured of this isomorphism without needing to know exactly which operators in these spaces are compact.

\section{EXAMPLE 5. Hankel operators of higher multiplicity.}

If $S$ is a unilateral shift of multiplicity $k$, where $k$ is any cardinal number, then an $S$-Hankel operator is an operator $H$ satisfying $S^{*} H=H S$.

Any $S$-Hankel operator can be represented by a matrix with operator entries $\left[A_{i j}\right]$ where $A_{i j}=B_{i+j}$, an operator on a $k$-dimensional Hilbert space. Then each operator $H$ in $\mathscr{J}\left(S, S^{*}\right)$ is the weak-operator limit of the bounded net of compact Hankel operators with matrices $\left[r^{i+j}\left(A_{i j}\right)_{r}\right], 0<r<1$. Thus $\mathscr{F}\left(S, S^{*}\right)$ is isomorphic to the second dual of its compact part.

\section{REFERENCES}

1. N. Bourbaki, Espace Vectoriels Topologiques, Hermann, Paris, 1955.

2. M. M. Day, Normed Linear Spaces, Academic Press, New York, 1962.

3. N. Dunford and J. T. Schwartz, Linear Operators, Volume I, Interscience Publishers, 1963.

4. R. N. Hevener, A functional analytic approach to Hankel and Toeplitz matrices, Thesis, University of Virginia, Charlottesville, 1965.

5. T. L. Kriete, III, B. Moore, III, and L. B. Page, Compact intertwining operators, Mich. J. Math., 18 (1971), 115-119. 
6. L. B. Page, Bounded and compact vectorial Hankel operators, Trans. Amer. Math. Soc., 150 (1970), 529-539.

7. R. Schatten, Norm Ideals of Completely Continuous Operators, Springer-Verlag. Berlin, 1960.

Received March 1, 1972 and in revised form February 26, 1973.

North Carolina State University 



\section{PACIFIC JOURNAL OF MATHEMATICS}

\section{EDITORS}

D. Gilbarg AND J. MILGRAM

Stanford University

Stanford, California 94305

\section{R. A. Beaumont}

University of Washington

Seattle, Washington 98105

\section{J. DUGUNDJI*}

Department of Mathematics University of Southern California Los Angeles, California 90007

RICHARD ARENS

University of California Los Angeles, California 90024

\section{ASSOCIATE EDITORS}
E. F. BECKENBACH
B. H. NEUMANN
F. WOLF
K. YoshidA

\section{SUPPORTING INSTITUTIONS}

\author{
UNIVERSITY OF BRITISH COLUMBIA \\ CALIFORNIA INSTITUTE OF TECHNOLOGY \\ UNIVERSITY OF CALIFORNIA \\ MONTANA STATE UNIVERSITY \\ UNIVERSITY OF NEVADA \\ NEW MEXICO STATE UNIVERSITY \\ OREGON STATE UNIVERSITY \\ UNIVERSITY OF OREGON \\ OSAKA UNIVERSITY
}

\author{
UNIVERSITY OF SOUTHERN CALIFORNIA \\ STANFORD UNIVERSITY \\ UNIVERSITY OF TOKYO \\ UNIVERSITY OF UTAH \\ WASHINGTON STATE UNIVERSITY \\ UNIVERSITY OF WASHINGTON \\ AMERICAN MATHEMATICAL SOCIETY \\ NAVAL WEAPONS CENTER
}

The Supporting Institutions listed above contribute to the cost of publication of this Journal, but they are not owners or publishers and have no responsibility for its content or policies.

Mathematical papers intended for publication in the Pacific Journal of Mathematics should be in typed form or offset-reproduced, (not dittoed), double spaced with large margins. Underline Greek letters in red, German in green, and script in blue. The first paragraph or two must be capable of being used separately as a synopsis of the entire paper. Items of the bibliography should not be cited there unless absolutely necessary, in which case they must be identified by author and Journal, rather than by item number. Manuscripts, in duplicate if possible, may be sent to any one of the four editors. Please classify according to the scheme of Math. Rev. Index to Vol. 39. All other communications to the editors should be addressed to the managing editor, Richard Arens, University of California, Los Angeles, California, 90024.

50 reprints are provided free for each article; additional copies may be obtained at cost in multiples of 50 .

The Pacific Journal of Mathematics is issued monthly as of January 1966. Regular subscription rate: $\$ 48.00$ a year (6 Vols., 12 issues). Special rate: $\$ 24.00$ a year to individual members of supporting institutions.

Subscriptions, orders for back numbers, and changes of address should be sent to Pacific Journal of Mathematics, 103 Highland Boulevard, Berkeley, California, 94708.

PUBLISHED BY PACIFIC JOURNAL OF MATHEMATICS, A NON-PROFIT CORPORATION

Printed at Kokusai Bunken Insatsusha (International Academic Printing Co., Ltd.), 270, 3-chome Totsuka-cho, Shinjuku-ku, Tokyo 160, Japan.

* C. DePrima will replace J. Dugundji until August 1974.

Copyright (C) 1973 by

Pacific Journal of Mathematics

All Rights Reserved 


\section{Pacific Journal of Mathematics}

\section{Vol. 47, No. $2 \quad$ February, 1973}

David Parham Bellamy, Composants of Hausdorff indecomposable continua; a mapping approach ........................ 303

Colin Bennett, A Hausdorff-Young theorem for rearrangement-invariant spaces ...........................................

Roger Daniel Bleier and Paul F. Conrad, The lattice of closed ideals and $a^{*}$-extensions of an abelian l-group ...

Ronald Elroy Bruck, Jr., Nonexpansive projections on subsets of Banach

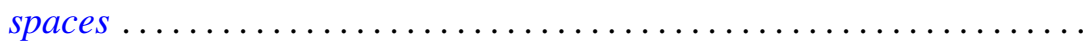

Robert C. Busby, Centralizers of twisted group algebras ............. 357

M. J. Canfell, Dimension theory in zero-set spaces ................ 393

John Dauns, One sided prime ideals ........................ 401

Charles F. Dunkl, Structure hypergroups for measure algebras . . . . . . . . . 413

Ronald Francis Gariepy, Geometric properties of Sobolev mappings ...... 427

Ralph Allen Gellar and Lavon Barry Page, A new look at some familiar spaces of intertwining operators ...........................

Dennis Michael Girard, The behavior of the norm of an automorphism of the

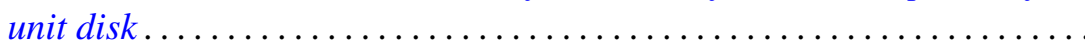

George Rudolph Gordh, Jr., Terminal subcontinua of hereditarily

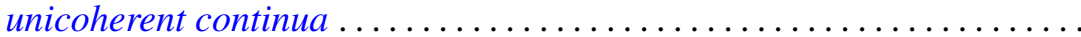

Joe Alston Guthrie, Mapping spaces and cs-networks. .

Neil Hindman, The product of $F$-spaces with $P$-spaces . 473

M. A. Labbé and John Wolfe, Isomorphic classes of the spaces $C_{\sigma}(S)$

Ernest A. Michael, On k-spaces, $k_{R}$-spaces and $k(X) \ldots$

Donald Steven Passman, Primitive group rings .

C. P. L. Rhodes, A note on primary decompositions of a pseudovaluation ...

Muril Lynn Robertson, A class of generalized functional differential equations

Ruth Silverman, Decomposition of plane convex sets. $I$.

Ernest Lester Stitzinger, On saturated formations of solvable Lie algebras................................

B. Andreas Troesch, Sloshing frequencies in a half-space by Kelvin inversion ...

L. E. Ward, Fixed point sets .

Michael John Westwater, Hilbert transforms, and a problem in scattering

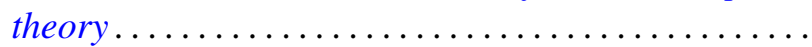

Misha Zafran, On the spectra of multipliers... 